\title{
An Electric Field Detector for high-performance measurements of the electric field in the ionosphere
}

D. Badoni ${ }^{* a b}$, G. Masciantonio ${ }^{a c}$, P. Cipollone ${ }^{b}$, G. Vannaroni $^{d e}$, P. Diego ${ }^{c d}$, R. Ammendola $^{b}$, V.A. Belyaev $f$, O. Simonellig for CSES-LIMADOU Collaboration

a Department of Physics, University of Rome Tor Vergata, Via della Ricerca Scientifica 1, I-00133 Rome, Italy

${ }^{b}$ INFN, Sez. of Rome Tor Vergata, Via della Ricerca Scientifica 1, I-00133 Rome, Italy.

${ }^{c}$ Department of Physics, University of Trento, 38123 Povo (TN), Italy

d INAF/IAPS, Roma Via Fosso del Cavaliere 100, I-00133 Rome, Italy

${ }^{e}$ Università Telematica Internazionale Uninettuno, Corso Vittorio Emanuele II 39, 00186 Rome, Italy

${ }^{f}$ National Research Nuclear University MEPhI, Moscow, Russia

${ }^{f}$ Innovation Design S.r.l., Via Libero Leonardi 4, 00173 Rome, Italy

E-mail:davide.badoni@roma2.infn.it,

giuseppe.masciantonio@roma2.infn.it,

piero.cipollonelroma2.infn.it, giuliano.vannaroniliaps.inaf.it,

piero.diego@iaps.inaf.it, roberto.ammendola@roma2.infn.it,

bz175@yandex.ru, osvaldo.simonelli@innodesi.com

An Electric Field Detector (EFD) for space applications has been designed and built in the framework of the CSES (China Seismo-Electromagnetic Satellite) mission. The instrument has been conceived for space-borne measurements of electromagnetic phenomena such as seimo-electromagnetic perturbations and more in general to investigate lithosphere- atmosphereionosphere EM coupling. The EFD consists of four probes designed to be installed on booms deployed from a 3-axes stabilized satellite. It measures electric field in a wide band of frequencies extending from quasi-DC up to about $5 \mathrm{MHz}$ subdivided in four frequency bands by a signal processing unit, with a resolution of the order of $1 \mu \mathrm{V} / \mathrm{m}$ with a wide dynamic range up to 120 $\mathrm{dB}$ in the lower DC-ULF band. The resolution is 40 times better than any other recent instrument of similar feature. With these characteristics, the described EFD represents the most performing and updated device so far developed for electric field measurements in near-space applications. The detector has been tested in laboratory both in a Faraday cage and in a Plasma Chamber that simulates the real ionospheric conditions. Topic of this paper is the technical description of the $\mathrm{EFD}$, its main characteristics and the test results.

The 34th International Cosmic Ray Conference,

30 July- 6 August, 2015

The Hague, The Netherlands

\footnotetext{
${ }^{*}$ Speaker.
} 


\section{Introduction}

We have designed and realized a new electric field detector (EFD) to be installed on board scientific space missions suitable to investigate electromagnetic phenomena in ionosphere. The instrument measures electric field in a large bandwidth from quasi-DC up to about $5 \mathrm{MHz}$. Similar instrument (ICE, [1]) was in operation for more than 5 years from June 2004 on-board DEMETER spacecraft for seismic activity detection. The resolution of the proposed EFD in the ULF band is better than $1 \mu \mathrm{V} / \mathrm{m}$ with a dynamic range of $120 \mathrm{~dB}$. The sensitivities in the other bands ( $E L F, V L F$ and $H F$ ) are better than $300 \frac{n V}{\sqrt{H z}}$ which, considering the boom lengths can be expressed in terms of electric field and becomes of the order of $50 \frac{n V}{\sqrt{H z} m}$ In this paper we present the description of the instrument electronics and the results of the preliminary tests performed on the EFD prototype in laboratory.

The general EFD block diagram is shown in fig. 1.

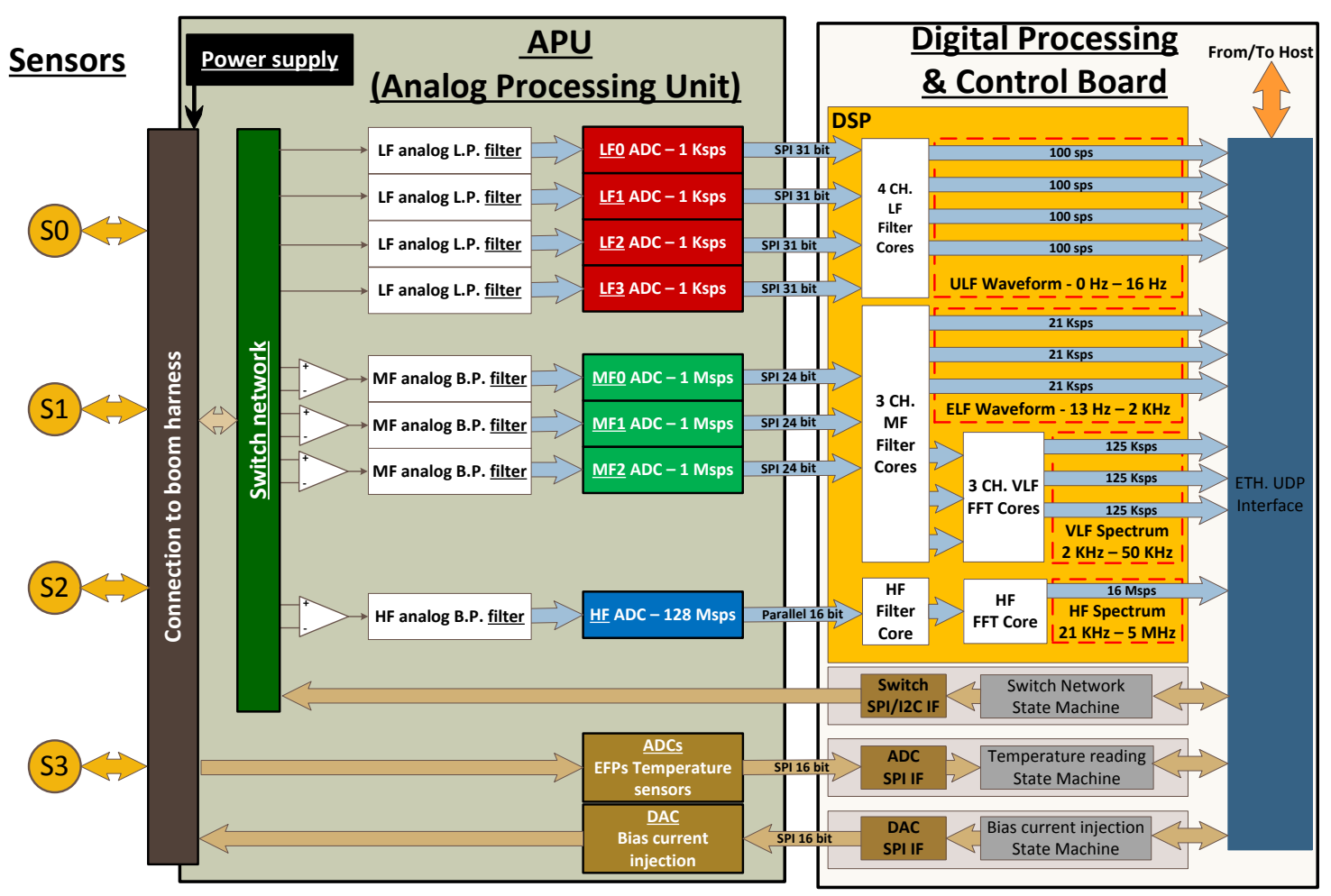

Figure 1: The EFD complete diagram..

The instrument consists of four independent identical sensors (from $S 0$ through $S 3$ ) followed by two boards for signal conditioning, digital conversion and processing. The sensors are installed at the tips of four booms (about four meters long) deployed from a 3-axes stabilized spacecraft. The Analog Processing Unit (APU) performs a fully flexible selection of the sensors and provides a preliminary filtering and band subdivision. The Digital Processing \& Control Board carries out a decimation process for $U L F$ and $E L F$ bands, the Fast Fourier Transform for $V L S$ and $H F$ bands and transmission control of data \& commands to/from host PC and APU. Data are presented in terms of waveforms for $U L F$ and $E L F$ bands and as signal spectra for the $V L F$ and $H F$. 
The EFD, equipped with only one pair of sensors, has been used in a Faraday Cage for a measurement champaign aimed at evaluating the electronic specifications and, preliminary, in a plasma chamber to verify the plasma compatibility.

\section{Description}

\section{Description of the sensors}

The main objective of the sensor is the measurements of local plasma potential at the tip of the boom. The electric field will be then derived by dividing the potential difference measured across a pairs of sensors to their mutual distance. Each sensor consists of a spherical electrode exposed to the ionospheric plasma and is connected to a very high input impedance voltage follower.

As well known, the voltage of an electrode in plasma is a function of the collected current which is the algebraic sum of the electron, ion and photoelectron components which, in turn, are determined by the so-called current/voltage characteristic. Along the characteristic curve we may define the contact impedance $(d V / d I)$ which is strongly varying along the curve. In particular, the contact impedance between electrode and plasma reaches a minimum when the electrode is biased at the plasma potential (assumed equal to zero) whereas significantly increases when the electrode acquires a negative potential. Usually a floating electrode in plasma acquires a negative potential (floating potential), thus the EFD has to use a current generator in order to bias itself close to the plasma potential to minimize the contact impedance and improve the measurement accuracy. The block diagram of one EFD sensor is shown in fig. 2 along with photograps of the prototype. Both voltage follower and current generator are embedded within the sphere. The electronics is shielded from the external current collecting electrode through an inner conductive shell which is bootstrapped at sensor potential in order to minimize the effect of the parasitic capacitance and thus to increase the frequency response. The short stubs placed at the opposite sides of the EFD (aligned with the boom axis) are aimed at reducing the asymmetry introduced by the presence of the boom and are bootstrapped at the same potential of the sensor. The lowpass filter visible in the shield bootstrap circuit is used to optimize the frequency response of the amplifier.

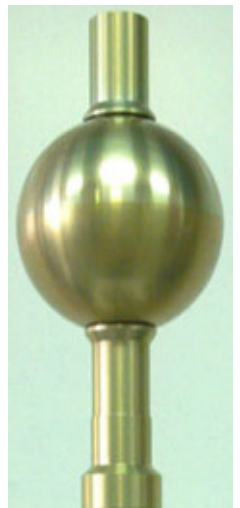

(a)

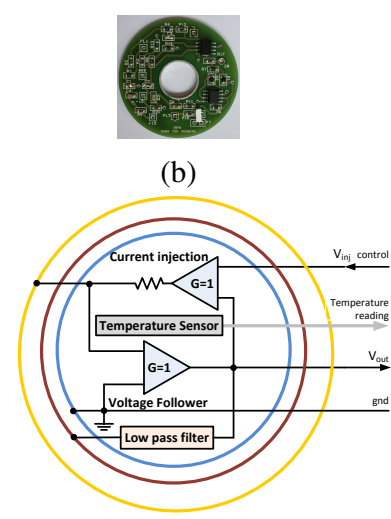

(c)

Figure 2: EFD probe prototype. Photograph of the sensor (a) and of the board (b). (c) Block diagram: orange sphere is the sensor current collector, brown is the inner bootstrapped shield and blue is the grounded shield.

\section{Description of the Analog Processing Unit (APU) and Digital Processing \& Control Board (DPCB)}

The main objective of $A P U$, whose architecture is depicted in fig. 1 is the subdivision of the signals coming from the sensors into three bands: $L F$ from 0 to $16 \mathrm{~Hz} ; \mathrm{MF}$ from $13 \mathrm{~Hz}$ to $50 \mathrm{kHz}$ 
and $H F$ from $21 \mathrm{kHz}$ to $5 \mathrm{MHz}$. We have four single channels for the $L F$ band which are obtained through low-pass third order Butterworth filters implemented with Sallen-Key architecture. Each sensor can be directly connected to its own channel. Output signals is digitized with 31 bit $A D C$ at $1 K s p s$ data rate. For the $M F$ band there are three independent channels consisting of a second order Butterworth pass-band filters implemented with a multiple feed-back architecture. The output signal is sampled with 24 bit $A D C s$ at $1 M s p s$ data rate. The $H F$ consists of a cascade of a pair of four order Butterworth high-pass and low-pass filters implemented with a Sallen-Key architecture. The $H F$ signal output is sampled with 16 bit $A D C s$ at $128 \mathrm{Msps}$ data rate. Note that the input of the $M F$ and $H F$ channels can be connected to the sensor through a switch matrix allowing a complete flexible reconfigurability. Moreover the APU contains the DAC needed to command the suitable bias current for the sensors.

The $D P C B$, described in fig. 1, provides a further digital elaboration on the APU output data ( $D S P$ block) in order to: i) improve the filter roll-off, ii) separate the $M F$ bands into $E L F$ and $V L F$ bands, iii) built the $F F T$ spectra of the $V L F$ and $H F$ bands. See table 1 for details. Moreover the $D P C B$ implements the digital stimuli for the bias current and the ethernet protocols for connection and data exchange to a host computer.

\begin{tabular}{lcccc}
\hline \multirow{2}{*}{ Name } & $\begin{array}{c}\text { APU } \\
\text { Freq. range }\end{array}$ & Name & $\begin{array}{c}\text { DPCB } \\
\text { Freq. range }\end{array}$ & OUTPUT \\
\hline LF & $0-16 \mathrm{~Hz}$ & ULF & $0-16 \mathrm{~Hz}$ & Waveform \\
& & ELF & $13 \mathrm{~Hz}-2 \mathrm{kHz}$ & Waveform \\
MF & $13 \mathrm{~Hz}-50 \mathrm{kHz}$ & VLF & $1 \mathrm{kHz}-50 \mathrm{kHz}$ & Spectrum \\
HF & $21 \mathrm{kHz}-5 \mathrm{MHz}$ & HF & $21 \mathrm{kHz}-5 \mathrm{MHz}$ & Spectrum \\
\hline
\end{tabular}

Table 1: Definition of the frequencies bands.

\section{Test results}

In order to verify the specifications and performance of the instrument several tests were planned and carried out on a prototype of the EFD limited to only 2 probes but with a complete DAQ system for signal filtering, acquisition and data processing. Fig. 3 shows the setup. One of the distinguishing features of the instrument is its high

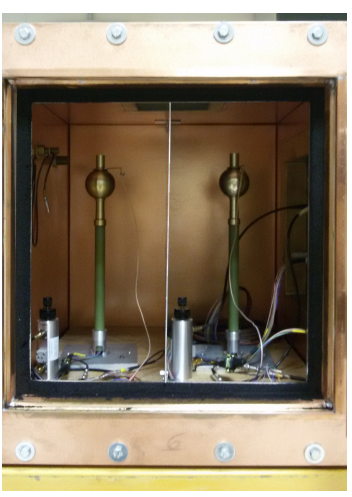

Figure 3: The experimental setup of the EFD installed in the Faraday cage resolution and sensitivity. The total output noise is the main feature that determines these parameters. It is directly related to the minimum detectable signal and therefore also to the obtainable "dynamic range". For the low noise measurements and for all other tests we have organized and realized a specific system of measurement instrumentations. Due to the high input impedance of the sensors a Faraday cage has been used to accommodate the sensors in order to shield them from the external electromagnetic environment and minimize the external interferences. The tests in Faraday Cage determine: Electronic noise, Dynamic range and Transfer function. The overall features of the EFD are the 
result of the design of both analog and digital chains. While for the digital processing it is easy to derive the actual performance directly from the component specifications (mainly those relevant to the ADCs), for the analog chain the complexity of the amplification and filter chains as well as the dependence on external parameters (e.g. coupling impedance input) requires accurate measurements. For these reasons all measurements were carried out taking the signals from the outputs of each analog processing chain (for each band) just at the ADCs inputs, prior digitization.

We have performed the tests varying two parameters: i) the equivalent electric circuit which represents the plasma coupling impedance and ii) the bias current. Only a representative subset of conditions have been chosen for each test. We have estimated the values of the plasma impedance for three different combinations of plasma densities and temperatures which can be considered as the extremes and medium of the values expected along the CSES orbit. The plasma coupling parameters adopted for measurements are shown in table 2 which represent the extreme and typical values of possible plasma conditions encountered along a ionospheric orbit [2].

\begin{tabular}{lccrc}
\hline \multirow{2}{*}{ Plasma } & \multicolumn{2}{c}{ Plasma conditions } & \multicolumn{2}{c}{ Impedance } \\
\cline { 2 - 5 } & Density & Temperature & Resistance & Capacitance \\
\hline$C_{1}$ : High Electron Temperature/Density & $10^{12} \mathrm{~m}^{-3}$ & $3000 \mathrm{~K}$ & $67 \mathrm{k} \Omega$ & $29 \mathrm{pF}$ \\
$C_{2}$ : Medium Electron Temperature/Density & $10^{10} \mathrm{~m}^{-3}$ & $2000 \mathrm{~K}$ & $660 \mathrm{k} \Omega$ & $6.6 \mathrm{pF}$ \\
$C_{3}$ : Low Electron Temperature/Density & $10^{9} \mathrm{~m}^{-3}$ & $1000 \mathrm{~K}$ & $970 \mathrm{k} \Omega$ & $4.8 \mathrm{pF}$ \\
\hline
\end{tabular}

Table 2: Plasma conditions in a ionospheric orbit and corresponding equivalent impedance values adopted for test measurements of the EFD prototype performance.

Electronic Noise The Electronic noise measurements are given in terms of Voltage Noise Spectral Density vs frequency as acquired by a Spectrum Analyzer and determine the EFD sensitivities in each band. In addition, for the $L F$ band the noise is given in terms of $V_{r m s}$ according to

$$
V_{r m s}=\sqrt{\int_{0}^{f_{h i g h}}\left(V_{D}(f)\right)^{2} d f}
$$

where $V_{D}(f)$ is the voltage noise density expressed in $\frac{V}{\sqrt{H z}}$ and $f_{\text {high }}$ is the high cutoff frequency of the LF band. The resulting $V_{r m s}$ determines the EFD resolution in that band.

Dynamic Range We define the Dynamic range as $D R=20 \cdot \log \frac{V_{R M S_{M A X}}}{V_{R M S_{M I N}}}$ where $V_{R M S_{M A X}}$ is the maximum $V_{R M S}$ obtainable at the output, in absence of distortions or saturations, measured for an injected sine wave whose frequency is centred at the middle of the band, whereas $V_{R M S_{M I N}}$ is:

- For the "LF" and "ELF" bands, the $V_{R M S}$ level of the noise measured close to the sine wave frequency, resulting from the integration of the voltage noise density in a $1 \mathrm{~Hz}$ bandwidth.

- For the "VLF" and "HF" bands, the $V_{R M S}$ level of the noise measured close to the sine wave frequency, resulting from the integration of the voltage noise density in a $\Delta F$ bandwidth, where $\Delta F$ is the frequency resolution of the $F F T$ processing.

Note that the analog $M F$ band is split in the two ELF and $V L F$ bands according to the digital processing defined in the general instrument description. 
Transfer Function The Transfer function between the EFD output and probe input is directly measured with a network analyzer.

\subsection{Noise test results}

Fig. 4 shows the voltage noise spectral densities determined for the $L F, M F$ and $H F$ channels measured in various plasma conditions. Panel (a) and (b) show the $L F$ noise density for $C_{2}$ and $C_{3}$ plasma conditions, respectively. Panel (c) gives the noise measured for $M F$ band under $C_{2}$ plasma condition and panel (d) provides the noise measured for $H F$ band for $C_{2}$ plasma condition.

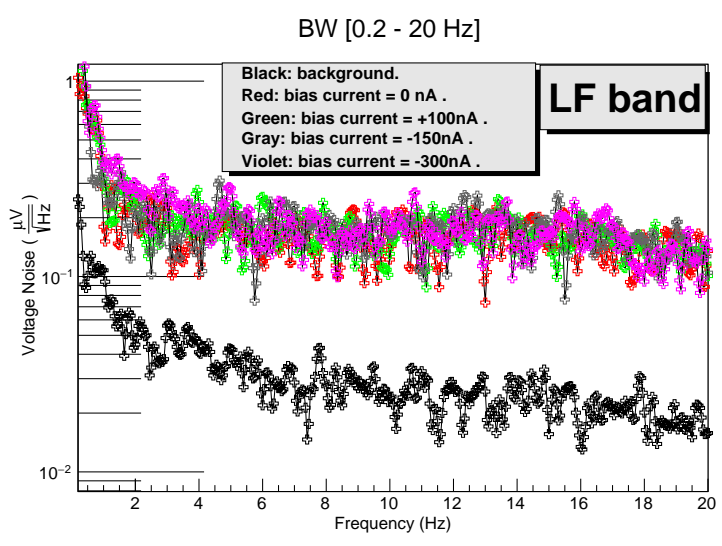

(a) LF band. $C_{2}$ plasma condition.

BW $[3-100 \mathrm{KHz}]$

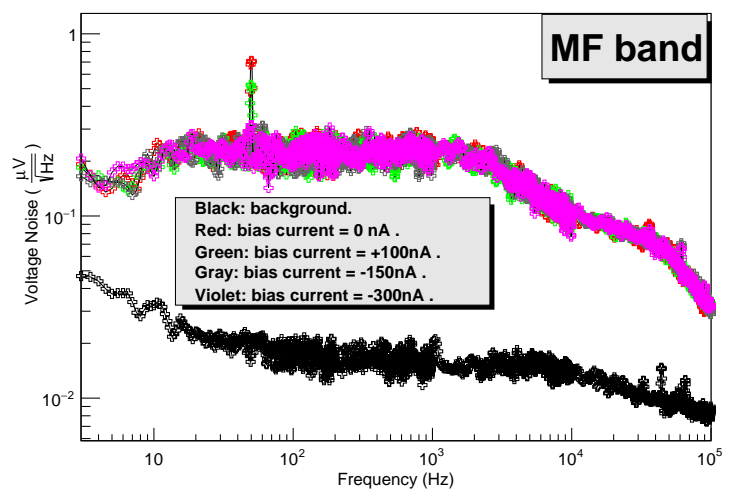

(c) MF band. $C_{2}$ plasma condition.

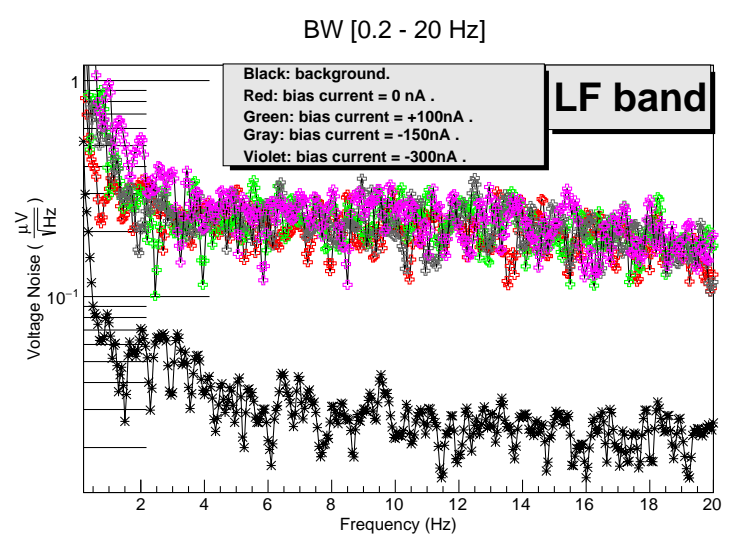

(b) LF band. $C_{3}$ plasma condition.

BW $[1 \mathrm{KHz}-15 \mathrm{MHz}]$

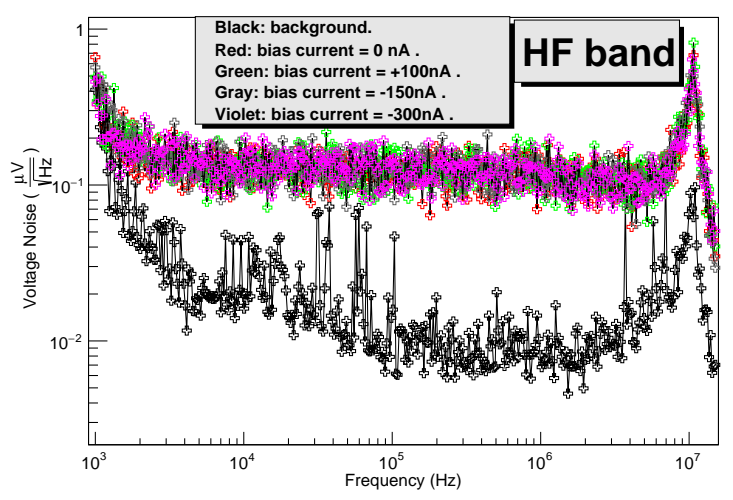

(d) HF band. $C_{2}$ plasma condition.

Figure 4: Voltage noise spectral density vs frequency for: (a) $L F$ channel with input set to $C_{2}$ plasma condition (medium electron temperatures/densities); (b) $L F$ channel with input set to $C_{3}$ plasma condition (low electron temperature/density); (c) $M F$ channel and (d) $H F$ channel both with input set to $C_{2}$ plasma condition. All tests have been obtained for several values of the bias current. The black traces represent the noise due to only the measurement instrumentation, while the EFD electronics is powered off.

The black traces in the various panels of fig. 4 represent the noise due to only the measurement instrumentation (spectrum analyzer), while the EFD electronics is powered off. All other traces 
correspond to measurements performed with the different values of bias current.

Table 3 shows the results of the calculated $V_{r m s}$ noise for various bias currents obtained with different plasma conditions applying eq. 3.1. Note that the noise measured for $L F$ under plasma

\begin{tabular}{lccc}
\hline Configuration & \multicolumn{3}{c}{ Plasma condition } \\
& $C_{1}$ & $C_{2}$ & $C_{3}$ \\
\hline Background & $0.20 \mu V_{r m s}$ & $0.19 \mu V_{r m s}$ & $0.22 \mu V_{r m s}$ \\
Bias current $=0 n A$ & $0.60 \mu V_{r m s}$ & $0.96 \mu V_{r m s}$ & $1.14 \mu V_{r m s}$ \\
Bias current $=100 n A$ & $0.65 \mu V_{r m s}$ & $1.16 \mu V_{r m s}$ & $1.30 \mu V_{r m s}$ \\
Bias current $=-150 n A$ & $0.72 \mu V_{r m s}$ & $1.21 \mu V_{r m s}$ & $1.42 \mu V_{r m s}$ \\
Bias current $=-300 n A$ & $0.58 \mu V_{r m s}$ & $1.59 \mu V_{r m s}$ & $1.66 \mu V_{r m s}$ \\
\hline
\end{tabular}

Table 3: $V_{r m s}$ noise for $L F$ band obtained by applying the eq. 3.1

condition $C_{3}$ is slightly worse then the one measured with plasma condition $C_{2}$. We have not shown the results obtained with the remaining plasma conditions $C_{1}$ as the noise spectral density in $L F$ is, in that case, significantly lower. The results also show that the noise spectral density is almost independent from the values of the injected bias current. On the basis of the CSES configuration which foresees boom lengths of about four meters (about eight meters tip to tip) the electric field resolution is better than $1 \mu \mathrm{V} / \mathrm{m}$.

\subsection{Dynamic range test results}

According to the definition of the analog chain dynamic range given above we obtain the values shown in table 4.

\begin{tabular}{lrr}
\hline Band & Analog chain & ADC \\
\hline LF & $\mathbf{1 2 0} \mathrm{dB}$ & $124 \mathrm{~dB}$ \\
ELF & $\mathbf{1 3 9} \mathrm{dB}$ & $105 \mathrm{~dB}$ \\
VLF & $\mathbf{1 1 5} \mathrm{dB}$ & $105 \mathrm{~dB}$ \\
HF & $\mathbf{8 7} \mathrm{dB}$ & $78 \mathrm{~dB}$ \\
\hline
\end{tabular}

Table 4: EFD: Dynamic range for each band of the analog chain and ADCs.

We obtain $120 d B$ for the $L F$ band, $139 d B$ for $E L F, 115 d B$ for $V L F$ and $87 d B$ for $H F$. Along with these data we have also shown the dynamic ranges quoted from manufacturer data-sheet for the various ADCs used for the different bands. As we can see, in several bands, the dynamic ranges of ADC are worse than the ones computed for the analog chains. In those cases the actual dynamic range of the overall system is limited by the ADCs performance.

\subsection{Transfer function test results}

Fig. 5 shows the analog transfer functions for $L F$ (panel (a)), $M F$ (panel (b)) and $H F$ (panel (c)). We have varied the bias current as indicated in the plot legend. As previously specified, the $M F$ band (panels (b)) includes both $E L F$ and $V L F$. Note that the slight decay of the transfer function amplitudes within the bandwidth of the $M F$ and $H F$ filters, starting at about $20 \mathrm{kHz}$, are due to the low-pass pole associated to the plasma coupling equivalent electric circuit. 

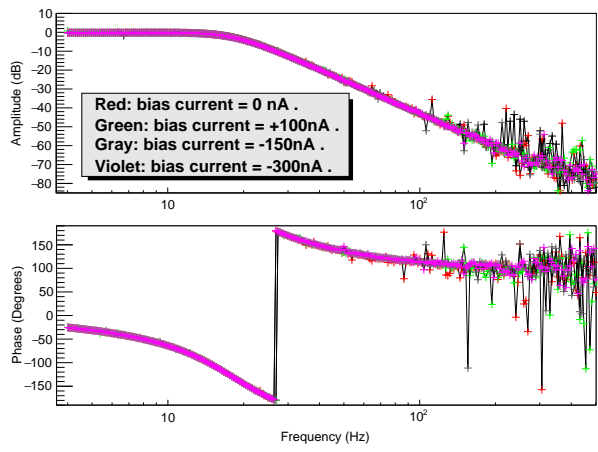

(a)
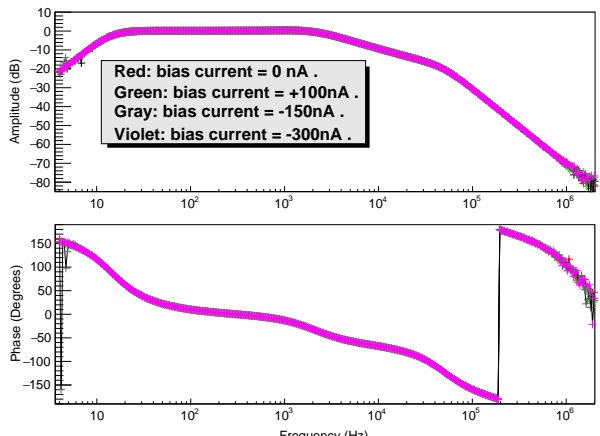

(b)
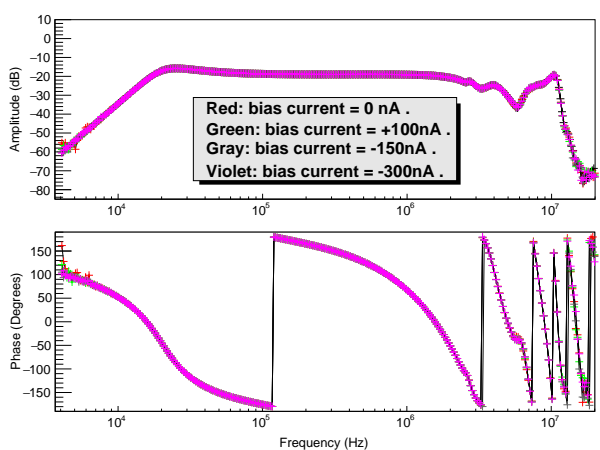

(c)

Figure 5: EFD transfer functions measured for the $L F$ (panel a), $M F$ (panel b) and $H F$ (panel c) bands with the equivalent electric circuit relevant to the medium plasma condition $C_{2}$ applied at the sensor input.

\section{Acknowledgments}

This work is financially supported by the Italian Space Agency (ASI) in the frame of the "Progetto Premiale Limadou".

The authors want to thanks the Chinese collegues from Lanzhou Institute of Physics (LIP China) who partecipate to the definition of the EFD Project and in particular Ma Mianjun, Lei Jungang and Cui Yang.

\section{References}

[1] J.J. Berthelier et al., ICE, the electric field experiment on DEMETER, Planet Space Sci., 54 pp.456-471, 2006.

[2] G. Vannaroni, Considerations on design of the front-end electronics, private comunication - May $6^{\text {th }}$ 2011 giuliano.vannaroni@iaps.inaf.it.

[3] A.V. Oppenheim, R.W. Shafer, Digital signal processing Prentice Hall, (1975).

[4] L.P. Huelsman, Active and passive Analog Filter Design Paperback, International Edition (1993). 